\title{
Viscous effects in planar magnetic X-point reconnection ${ }^{\star}$
}

\author{
I. J. D. Craig ${ }^{1}$, Y. E. Litvinenko², and T. Senanayake ${ }^{1}$ \\ 1 Department of Mathematics, University of Waikato, Private Bag 3105, Hamilton, New Zealand \\ e-mail: sts1@math.waikato.ac.nz \\ 2 Institute for the Study of Earth, Oceans, and Space, University of New Hampshire, Durham, NH 03824, USA
}

Received 1 September 2004 / Accepted 15 December 2004

\begin{abstract}
The impact of viscous dissipation is considered on magnetic reconnection in closed line-tied magnetic $X$-points. It is shown that viscous effects can provide fast energy dissipation for disturbances which do not alter the initial $X$-point topology. If the $X$-point topology is altered, then the rate of viscous dissipation depends on both the perturbed topology and the relative magnitudes of viscosity and electric resistivity. New solutions are demonstrated, which derive from the combination of resistive and viscous effects. The solutions are characterized by monotonically decaying modes which are qualitatively different from the previously known oscillatory modes in nonviscous resistive $X$-point reconnection. These results suggest that viscous heating in magnetic $X$-points may be an important effect in solar flares.
\end{abstract}

Key words. magnetohydrodynamics (MHD) - Sun: flares

\section{Introduction}

Magnetic reconnection is believed to be responsible for the rapid energy release in solar and stellar flares. Although exact descriptions of magnetic reconnection are difficult to obtain, an analytic solution can be derived for the case of small disturbances of line-tied magnetic $X$-points in planar geometries when the plasma pressure is small enough. This solution has application to low beta plasmas such as the solar corona. Specifically, Craig \& McClymont (1991, 1993) and Hassam (1992) used an eigenfunction description to show that the reconnection rate is "fast", in the sense that the energy dissipation rate depends only logarithmically on the plasma resistivity $\eta$. The classical value of $\eta$ in the corona is of order $10^{-12}$ or smaller, as measured by the inverse Lundquist number, and this weak dependence is essential in providing significant energy release rates in flares.

The original studies of $X$-point reconnection have now been extended to include gas pressure, axial field components and plasma inertial effects (Ofman et al. 1993; McClymont \& Craig 1996; McClements et al. 2004). Recently McLaughlin \& Hood (2004) examined a related problem involving the propagation and refraction of wave-packets in the vicinity of a magnetic null (see also Craig \& Watson 1992; Hassam \& Lambert 1996). What seems to have been neglected so far are the effects of fluid viscosity. This neglect is surprising, given that the leading term

^ This work was supported by NSF grant ATM-0136718, NASA grant NAG5-11797 and the New Zealand Marsden Fund. in the viscous stress tensor dominates the plasma resistivity by many orders of magnitude for typical coronal plasmas, a point emphasized in a theoretical context by Hollweg (1986).

It is interesting that viscous effects may also be implied by recent observational studies of solar flares (McKenzie \& Hudson 1999; McKenzie 2000; Asai et al. 2004). Although observed flare outflows provide strong evidence for reconnection, exhaust speeds are significantly lower than the Alfvénic outflows predicted by standard reconnection models. This effect would be consistent with the theoretically expected large viscosity, because viscosity is known to lead to slower inflows and outflows in steady state Sweet-Parker reconnection regime (Park et al. 1984). More detailed study of time-dependent $X$-point reconnection may therefore be of interest for solar flare modeling.

The purpose of this paper is to demonstrate that viscosity can indeed have a profound effect on the dynamics of magnetic reconnection at magnetic $X$-points. Although resistivity is required to account for the decay of the free magnetic energy, we point out that viscosity can dissipate not only the kinetic energy but also a significant fraction of the magnetic energy in a dynamically evolving plasma. We also demonstrate that the visco-resistive coupling produces significant new phenomena which could be important in the general context of coronal energy dissipation. The $X$-point relaxation problem is discussed in Sect. 2 where we present typical simulations showing the visco-resistive dynamics. We give a brief theoretical interpretation of our results in Sect. 3 and summarize in Sect. 4. 


\section{X-point reconnection dynamics}

\subsection{The initial $X$-point field}

We begin by introducing a flux function $\psi$ for the dimensionless planar magnetic field $\boldsymbol{B}=\nabla \psi \times \hat{z}$. For an initial potential field of the form $\boldsymbol{B}_{E}=(y, x)$, we have

$\psi_{E}=-\frac{1}{2} r^{2} \cos (2 \theta)=-\frac{1}{2}\left(x^{2}-y^{2}\right)$.

This field initially is immersed in a static, uniform-density plasma and anchored by perfectly conducting rigid boundary centered at the origin. We are interested in what happens when the initial $X$-point is perturbed by the superposition of a small magnetic perturbation. Figures $1 \mathrm{a}$ and $1 \mathrm{~b}$ show two perturbed $X$-point configurations. In Fig. 1a the disturbance field raises the magnetic energy of the $X$-point but maintains the topology inherent in the equilibrium field. By contrast, in Fig. 1b, the original flux function $\psi_{E}$ has been perturbed in a way that changes the topology by altering the separatrix angle of the initial field. As a result the excess flux in the left and right lobes has to be reconnected through the $X$-point before the equilibrium configuration can be regained.

It should be stressed that magnetic reconnection is the only mechanism which can alter the topology of the initial field. Viscous dissipation can remove kinetic energy from the fluid but, in the absence of resistive reconnection, any "topological component" in the initial disturbance cannot be removed.

\subsection{Linearized momentum and induction equations}

We consider the line-tied $X$-point problem as originally formulated by Craig \& McClymont (1991) but generalized to include the effects of a scalar fluid viscosity. The linearized MHD equations are written in non-dimensional form. We scale all quantities using the reference values of the magnetic field strength $B_{0}$, the number density $n_{0}$ and the length $\ell_{0}$. Typical coronal values $B_{0}=10^{2} \mathrm{G}, n_{0}=10^{9} \mathrm{~cm}^{-3}$, and $\ell_{0}=10^{9.5} \mathrm{~cm}$ identify the reference Alfvén speed $v_{\mathrm{A}} \simeq 10^{9} \mathrm{~cm} \mathrm{~s}^{-1}$ and the Alfvén time $\tau_{\mathrm{A}}=\ell_{0} / v_{\mathrm{A}} \simeq 3 \mathrm{~s}$.

Perturbations of the potential field $\psi_{E}$ are governed by the linearized momentum and induction equations for a compressible cold plasma with negligible gas pressure:

$\partial_{t} \boldsymbol{v}=-\nabla^{2} \psi \nabla \psi_{E}+v\left(\nabla^{2} \boldsymbol{v}+\frac{1}{3} \nabla \nabla \cdot v\right)$,

$\partial_{t} \psi=-\boldsymbol{v} \cdot \nabla \psi_{E}+\eta \nabla^{2} \psi$

Here and in what follows $\psi$ denotes the first-order perturbation of the flux function with respect to the equilibrium $\psi_{E}$, and $v$ denotes the first-order velocity of the fluid.

The dimensionless dissipation coefficients $v$ and $\eta$ are scaled by $v_{\mathrm{A}} \ell_{0}$ and $4 \pi v_{\mathrm{A}} \ell_{0} / c^{2}$, so that $\tau_{\mathrm{A}} / v$ is the global viscous diffusion time scale and $\tau_{\mathrm{A}} / \eta$ the resistive diffusion time scale of the background plasma. Adopting the coronal values given above and assuming a temperature of $10^{6} \mathrm{~K}$ gives the coefficients $v \simeq 10^{-4.5}$ and $\eta \simeq 10^{-14.5}$ (Spitzer 1962). These small numbers indicate that steep gradients must develop in

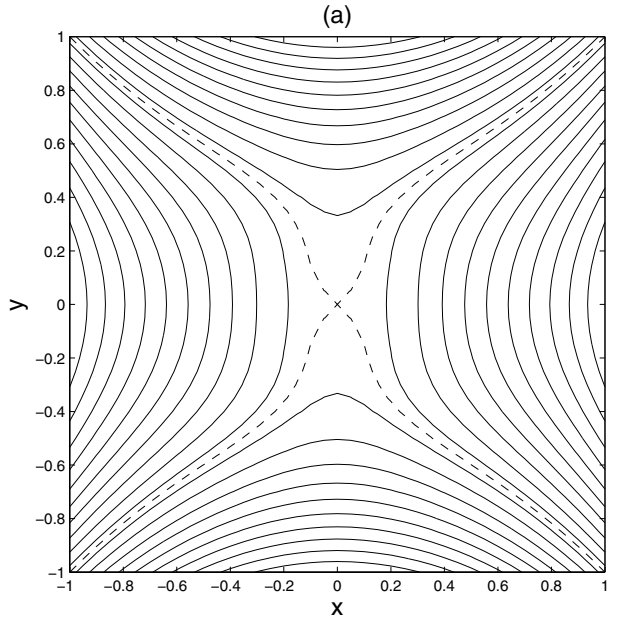

(b)

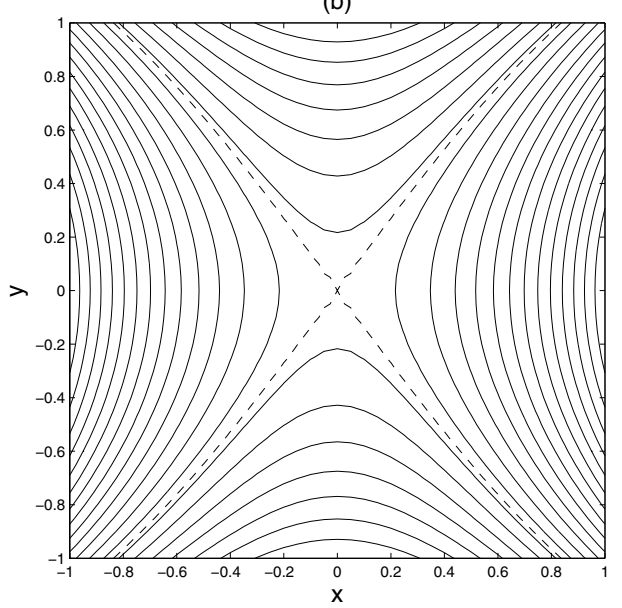

Fig. 1. Field lines of two perturbed $X$-point configurations. In a) the equilibrium flux function has been perturbed by a disturbance which maintains the original $X$-point topology. In b), however, the disturbance field has the effect of altering the connections between the equilibrium field lines.

the magnetic and velocity fields if significant dissipation is to occur. It is also clear that viscous effects can be of paramount importance in flare-related reconnection, given that $v \gg \eta$. One caveat is that the magnetic field should be weak enough at the reconnection site in order to justify the use of a scalar viscosity (Hollweg 1986).

\subsection{Global energy losses}

We are primarily concerned with the effects of viscous dissipation on the $X$-point energy losses. A convenient form for the global energy dissipation can be obtained by dotting the momentum equation with $\boldsymbol{v}$, and substituting for $\boldsymbol{v} \cdot \nabla \psi_{E}$ from the induction equation. An integration over the source volume, taking $\partial_{t} \psi=v=0$ on the surface bounding $V$, then yields

$\frac{\mathrm{d}}{\mathrm{d} t} \int \frac{1}{2}\left(v^{2}+B^{2}\right) \mathrm{d} V=$

$-\int\left(\eta\left(\nabla^{2} \psi\right)^{2}+v\left[(\nabla \times \boldsymbol{v})^{2}+\frac{4}{3}(\nabla \cdot \boldsymbol{v})^{2}\right]\right) \mathrm{d} V$ 

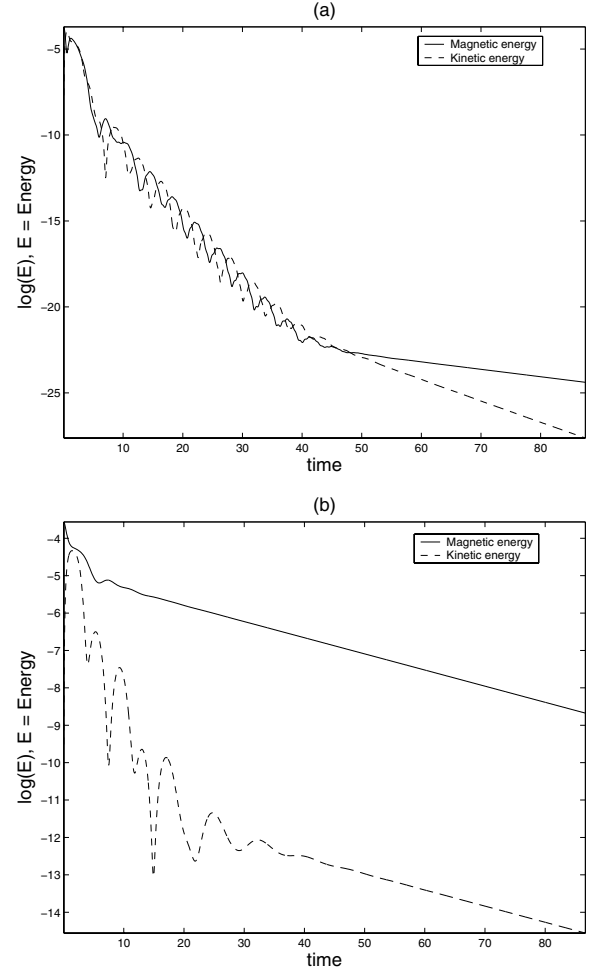

Fig. 2. The decay of the disturbance field energy for the two initial conditions of Fig. 1. The results show the spatially integrated kinetic and magnetic energies for the fluid for the cases $v=10^{-3}$ and $\eta=10^{-5}$. The reconnective disturbance of $\mathbf{b}$ ) is characterized by a relatively slow monotonic decay in the magnetic energy.

This expression makes explicit the resistive and viscous contributions to the decay of the total energy but does not isolate the separate magnetic and kinetic energy components.

In what follows we simulate the momentum and induction equations over the square $|x|,|y| \leq 1$ to determine the evolution of the disturbance fields $\psi(x, y, t)$ and $\boldsymbol{v}(x, y, t)$ with $\boldsymbol{v}=0$ initially. An explicit time-stepping scheme was adopted based on a square mesh whose step size $\Delta$ scales as $(\eta v)^{1 / 4}$ (see Sect. 3.2). The computations discussed below required step sizes $\Delta<10^{-2}$ to achieve satisfactory resolution.

To obtain insight into the physical processes involved, we study representative examples of the $X$-point dynamics for a fixed value of $v$ with different values of $\eta$. The global magnetic and kinetic energies were computed numerically using a second order numerical quadrature.

\subsection{Computed X-point solutions}

Figure 2 summarizes the decay of the disturbance field energy for the two initial conditions of Fig. 1. The results show the spatially integrated kinetic and magnetic energies for the fluid for the cases $v=10^{-3}$ and $\eta=10^{-5}$.

Figure $2 \mathrm{a}$ shows the non-reconnective mode with the initial conditions

$\psi(x, y, 0)=\left(1-x^{2}\right)\left(1-y^{2}\right) \mathrm{e}^{-s^{2}}$,

$s=\frac{1}{2} \log \left(x^{2}+y^{2}\right)$.
The initial rapid decline in energy $(t<10)$ is followed by a stage of fast oscillatory decay in which the kinetic and magnetic energies continually interchange $(10<t<40)$. The final phase is characterized by a slow decline involving the weak non-oscillatory separation of the kinetic and magnetic energy components of the plasma. These results - which are virtually unchanged by reductions in resistivity - make it clear that viscous damping is capable of removing the bulk of the free magnetic energy within several Alfvén times. In this respect viscous damping of non-reconnective perturbations is at least as effective as the well studied case of resistive damping (see Sect. 3.1 below).

The situation changes markedly when reconnective modes are considered. Figure $2 \mathrm{~b}$ shows the decay of the initial disturbance

$\psi(x, y, 0)=\left(1-x^{2}\right)\left(1-y^{2}\right)$.

Although roughly half the initial magnetic energy is rapidly transferred to the kinetic energy of the fluid for $t<2$, the later phase is characterized not by equipartition but by a rapid separation of kinetic and magnetic energy components. In particular, the slow monotonic decline of the magnetic energy contrasts with the much faster oscillatory decline of the global kinetic energy. As $\eta$ is reduced (with $v$ fixed), we find that the magnetic decline follows the decay law $\exp (-\gamma t)$ with $\gamma \simeq \eta^{1 / 2}$. The resulting energy separation reflects the fact that viscous damping cannot remove the topological energy of the disturbance. The separation is most apparent when $\eta \ll v$ but begins to break down when $\eta$ approaches $v$.

Figure 3 shows the behaviour of the reconnective perturbation when $v$ and $\eta$ are of the same order. The case $\eta=v$ appears to be unique in the sense that the solution is characterized by the slow monotonic decay of both the magnetic and kinetic energies, apart from oscillations with a very small amplitude in the magnetic energy (Fig. 3a). If the resistivity is increased further, $\eta>v$, oscillatory behaviour in the magnetic energy re-emerges (Fig. 3b). A separation of the kinetic and magnetic energies is present at large times $(t>30)$ when the global energy is essentially insignificant. However, in marked contrast to Fig. 2b, the bulk of the excess energy now resides in the kinetic, rather than the magnetic component of the total energy.

\section{Theoretical interpretation}

\subsection{Non-reconnective disturbances}

In the case of non-reconnective disturbances, viscous damping can be understood in much the same manner as resistive dissipation in nonviscous plasmas, for which there is a complete analytic theory (Craig \& McClymont 1991, 1993; Hassam 1992). The resistive theory is based on the existence of oscillatory cylindrical eigenfunction solutions which damp out at a rate $\alpha \simeq-\omega^{2} / 2$ determined by the fundamental oscillation frequency $\omega \simeq \pi /|\ln \eta|$ (Craig \& McClymont 1991). After the energy contained in the eigenmodes has been dissipated, all that remains is a slowly decaying, energetically insignificant "selfsimilar" mode, localized to the diffusion region (Hassam 1992; 


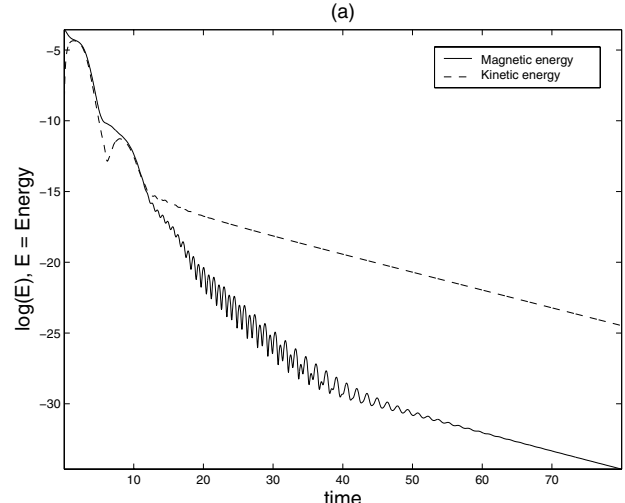

(b)

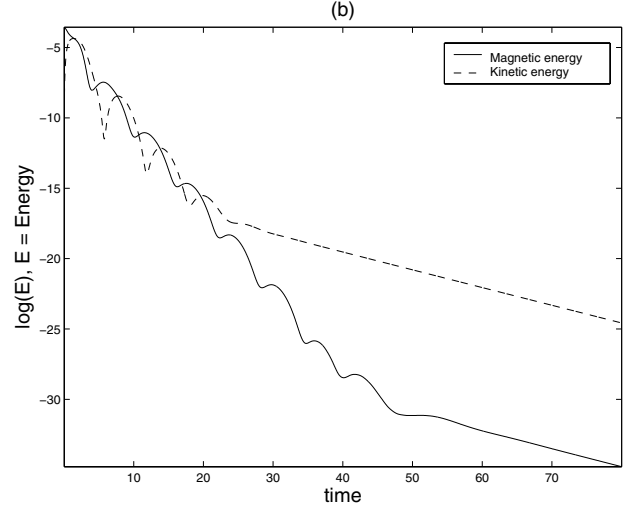

Fig. 3. a) shows the behaviour of the reconnective perturbation when $v$ and $\eta$ are of the same order $(\eta=v=0.001)$. Note that when the resistivity is increased beyond $v$ as in $\mathbf{b})(\eta=0.003, v=0.001)$ oscillatory behaviour in the magnetic energy re-emerges.

Craig \& McClymont 1993). This mode is obtained by assuming that the advection of the magnetic field can be balanced by resistive energy dissipation.

The key $\ln \eta$ dependence of the oscillatory decay can be understood by noting that resistivity introduces a small length scale $r=r_{\mathrm{s}}=\eta^{1 / 2}$ into the problem, which determines the size of the diffusion region surrounding the origin. The oscillation frequency is determined by the time it takes for a cylindrical wave launched from the outer boundary $r \simeq 1$ (idealized as a circle for analytic purposes) to reach the diffusion region:

$\frac{1}{\omega} \simeq \frac{2}{\pi} \int_{r_{\mathrm{s}}}^{1} \frac{\mathrm{d} r}{v_{\mathrm{A}}(r)} \simeq \frac{2}{\pi}\left|\ln r_{\mathrm{s}}\right|$,

where $v_{\mathrm{A}}=r$ is based on the equilibrium solution $\psi_{E}$. The global energy decays monotonically with the resistive damping rate $\alpha$ but there is a continual exchange of magnetic and kinetic energies on the oscillation period $2 \pi / \omega$.

Figure $2 \mathrm{a}$ shows that the energy losses are characterized by an oscillatory phase which sets in after the initial transients have died out. This behaviour can be understood if we assume that viscosity, by analogy with resistivity, introduces a viscous scale $r=r_{\mathrm{s}}=v^{1 / 2}$ into the problem, resistive effects being negligible in this case. The monotonic decay which sets in after the oscillatory modes have dissipated $(t>50)$ is then explained by balancing weak Lorentz forces against the viscous forces. This interpretation holds good for non-reconnective modes typified by Fig. 2a.

\subsection{Reconnective disturbances}

The arguments given above cannot explain the monotonic energy decline for reconnective disturbances, illustrated in Fig. 2b. An additional mechanism of energy dissipation is required to explain the simulation results. Below we argue that the simultaneous presence of viscosity and resistivity introduces a new length scale and indeed allows an extra channel of energy dissipation. This is because the viscous and resistive terms are capable of balancing the Lorentz force and advection, respectively.

Balancing the terms on the right hand sides of Eqs. (2) and (3) leads to the order-of-magnitude relationships:

$v r_{\mathrm{s}} \simeq \frac{\eta \psi}{r_{\mathrm{s}}^{2}}, \quad \frac{\psi}{r_{\mathrm{s}}} \simeq \frac{v v}{r_{\mathrm{s}}^{2}}$,

which give the new visco-resistive scale $r_{\mathrm{s}}=(\eta v)^{1 / 4}$. The emergence of this scale in viscous reconnection problems has been noticed by Park et al. (1984) and Hassam \& Lambert (1996). We expect this scale to develop when viscosity and resistivity act in unison to damp the oscillatory modes of the plasma and prevent energy equipartition in reconnective disturbances.

To understand how the emergence of the visco-resistive scale influences the dissipation of reconnective disturbances, we note that for $v \geq \eta$ the three dissipation length scales are ordered as follows:

$\eta^{1 / 2} \leq(\eta v)^{1 / 4} \leq v^{1 / 2}$

Fast oscillatory reconnection requires the development of strong localized currents on the smallest scale $r_{\mathrm{s}}=\eta^{1 / 2}$. When viscosity is present, however, the Lorentz force acting on the plasma can be diminished by viscous effects. For comparable magnitudes in the $\boldsymbol{B}$ and $\boldsymbol{v}$ fields, the ordering (9) shows that the viscous force can balance the Lorentz force on the viscoresistive scale, and thus undermine the conditions required for fast oscillatory reconnection. Equipartition now breaks down and there is a separation of the global kinetic and magnetic energies. In this case the global decay is limited by the damping of the magnetic field on the resistive time scale:

$\frac{\tau_{\eta}}{\tau_{\mathrm{A}}}=\frac{r_{\mathrm{s}}^{2}}{\eta}=\left(\frac{v}{\eta}\right)^{1 / 2}$,

where $r_{\mathrm{s}}=(\eta v)^{1 / 4}$.

An interesting limiting case is $\eta \rightarrow v$, when all the three dissipation length scales are the same. The case $\eta=v$ (Fig. 3a) evidently provides a transition between the regimes of fast oscillatory reconnection for $\eta>v$ (Fig. 3b) and slow monotonic decay of the magnetic energy $\sim \exp (-\gamma t)$. In the latter case, simulations show that the decay time of the magnetic energy scales as $\gamma^{-1} \simeq \eta^{-1 / 2}$. This is consistent with the theoretical prediction for $\tau_{\eta}$ with a fixed $v$.

\subsection{Summary}

In the case of non-reconnective disturbances our results imply that viscosity, in common with resistivity, can provide fast oscillatory dissipation of the excess magnetic energy, and the 
dissipation rate only logarithmically depends on the magnitude of the dissipative coefficient. The problem is dominated by the viscous length scale $v^{1 / 2}$ in the case $\eta<v$ and by the resisitve length scale $\eta^{1 / 2}$ in the case $\eta>v$. The oscillatory behaviour of the solution is not sensitive to the relative magnitudes of the viscous and resistive coefficients.

In the case of reconnective (topology-altering) disturbances, however, we find that fast oscillatory reconnection can occur only if $\eta>v$. When this condition is not met, oscillatory wave modes are effectively suppressed, and the velocity and magnetic field amplitudes are no longer coupled via equipartition. Thus for $\eta \leq v$, oscillatory damping is replaced by monotonic decay in which the global energy resides mainly in the magnetic field. This behaviour can be understood in terms of the emergence of a visco-resistive length scale $r_{\mathrm{s}}=(\eta v)^{1 / 4}$. Notably, the development of monotonically decaying solutions is not possible if either viscosity or resistivity is neglected.

\section{Discussion}

We have seen that the presence of viscosity can dramatically affect the evolution of resistive $X$-point disturbances. For non-reconnective disturbances, well-defined oscillatory eigenmodes develop which lead to rapid energy dissipation. In the case of reconnective disturbances, however, the magnitude of the viscous term does become critical. For $\eta \leq v$, oscillatory reconnection is suppressed and the global magnetic energy decays monotonically at a significantly slower rate than the kinetic energy. This behaviour is associated with the emergence of the visco-resistive scale $r_{\mathrm{s}}=(\eta v)^{1 / 4}$. Even though the possibility of fast reconnection is lost, a significant fraction of the disturbance field energy is rapidly damped by viscosity on an Alfvénic time scale. For $\eta>v$ fast oscillatory reconnection is regained.

Implications of these results for magnetic energy release in solar flares are very promising. Our exploratory analysis suggest that viscosity is very efficient in coronal magnetic $X$-points for which $v \gg \eta$. Since roughly half of the energy associated with any disturbance is expected to be transferred into the motion of the fluid, viscosity should be effective in removing a significant fraction of the disturbance field energy within a few Alfvén times, thus significantly contributing to the overall flare energy budget.
We stress that the role of viscosity in $X$-point reconnection is nontrivial. On one hand, viscous effects enhance the energy dissipation rate initially, "on a first bounce". Viscous dissipation operates on the length scale $v^{1 / 2}$, leading to the decay time $\left(v^{1 / 2}\right)^{2} \tau_{\mathrm{A}} / v=\tau_{\mathrm{A}}$. Thus a significant fraction (roughly a half) of the initial disturbance energy will be transferred to the fluid motion and dissipated by viscosity within a few Alfvén times, which is less than a minute for the illustrative numerical values assumed in Sect. 2.2. On the other hand, viscosity slows down the dissipation rate at later times. The dissipation time scale for the remaining energy in the system is of order $\tau_{\eta}$, which is a few days for the same numerical values.

Clearly further studies of viscous magnetic reconnection in the solar corona are warranted. Of particular theoretical and practical interest is the extension of our analysis to the parameter regime in which the magnetic field is sufficiently strong at the reconnection site. Then a single scalar viscosity coefficient can no longer be adopted, and the Braginskii coefficients have to be used to calculate the viscous stress tensor (Hollweg 1986).

Acknowledgements. Discussions with Gabriel Fruit have been much appreciated.

\section{References}

Asai, A., Yokoyama, T., Shimojo, M., \& Shibata, K. 2004, ApJ, 605, L77

Craig, I. J. D., \& McClymont, A. N. 1991, ApJ, 371, L41

Craig, I. J. D., \& McClymont, A. N. 1993, ApJ, 405, 207

Craig, I. J. D., \& Watson, P. G. 1992, ApJ, 393, 385

Hassam, A. B. 1992, ApJ, 399, 159

Hassam, A. B., \& Lambert, R. P. 1996, ApJ, 472, 832

Hollweg, J. V. 1986, ApJ, 306, 730

Ofman, L., Morrison, P. J., \& Steinolfson, R. S. 1993, ApJ, 417, 748

McClements, K. G., Thyagaraja, A., Ben Ayed, N., \& Fletcher, L. 2004, ApJ, 609, 423

McClymont, A. N., \& Craig, I. J. D. 1996, ApJ, 466, 487

McKenzie, D. E. 2000, Sol. Phys., 195, 381

McKenzie, D. E., \& Hudson, H. S. 1999, ApJ, 519, L93

McLaughlin, J. A., \& Hood, A. W. 2004, A\&A, 420, 1129

Park, W., Monticello, D. A., \& White, R. B. 1984, Phys. Fluids, 27, 137

Spitzer, L. 1962, Physics of fully ionized gases (John Wiley \& Sons) 